\title{
ALGEBRAS OF RIEMANN INTEGRABLE FUNCTIONS
}

\author{
K. G. JOHNSON
}

In the present paper we shall consider from two different standpoints the real Riemann integrable functions on a bounded closed interval $I$ contained in $E^{n}$. Our results will be extendable to the complex case by standard arguments.

(1) In $\S 1$ we designate the real valued Riemann integrable functions by $R(I)$. If $R(I)$ is normed by $\|f\|=\sup _{x \in I}|f(x)|$ then it is known that $R(I)$ is a Banach algebra. We show that $R(I)$ can be characterized as a $B(S, \Sigma)$ space [1] which consists of all uniform limits of real finite linear combinations of characteristic functions of sets in $I$ having Jordan content. This characterization immediately yields a representation of the dual space of $R(I)$.

(2) We consider in $\$ 2$ a collection of equivalence classes of functions in $R(I)$ : we define $R_{\infty}(I)$ to be the collection of classes such that $f_{1}$ and $f_{2}$ are in the same class if and only if $f_{1}-f_{2}$ vanishes everywhere except on a set of Lebesgue measure zero. If $[f]$ is an element of $R_{\infty}(I)$ we define the norm $\|[f]\|$ as follows. Let $N$ designate the subsets of $I$ of Lebesgue measure zero. Then if $f \in[f]$ let $\|[f]\|$ $=\inf _{S \in N} \sup _{x \notin S}|f(x)|=\|f\|_{\infty}$. It is obvious that this norm is induced by the norm of $L_{\infty}(I)$. It is also clear that $R_{\infty}(I)$ is isometrically isomorphic to a normed subalgebra of $L_{\infty}(I)$. Designating the Boolean ring of null Jordan sets by $\mathcal{I} \cap N$, we show that $R_{\infty}(I)$ $=R(I) / B(I, \mathrm{~g} \cap N)$ after first establishing that $B(I, g \cap N)$ (interpreted in the $B(S, \Sigma)$ sense) is a closed ideal in $R(I)$.

It is a pleasure to acknowledge the advice of Professor Fred B. Wright and Professor F. Quigley.

1. The algebra $R(I)$.

Definition 1.1. Let $I$ be a bounded and closed interval in $E^{n}$. The Jordan sets in $I$ are those subsets of $I$ whose boundaries have Lebesgue measure zero. We will designate the collection of Jordan sets by $\mathcal{d}$.

The following lemma is well known.

LEMma 1.2. With the set theoretic operations $\cup, \cap$ and complementation, $\mathrm{g}$ is a Boolean algebra.

LeMmA 1.3. Let $v$ be a collection of pairwise disjoint open cubes in I such that if $C=\bigcup\{v: v \in V\}$ then $I-C$ has Lebesgue measure zero. Let

Received by the editors March 27, 1961 and, in revised form, May 3, 1961. 
$V_{L}$ be a subset of $V$ and let $S=\cup\left\{v: v \in V_{L}\right\}$. Then $S$ is a Jordan set.

Proof. We will show that the boundary of $S$ is contained in $I-C$ and thus has measure zero. Now $S$ is open so its boundary is contained in $I-S$. Let $x$ be in $C-S$. Then $x$ is in a $v$ such that $v \cap S=\varnothing$ because the $v$ in $v$ are pairwise disjoint. Hence $x$ is not in the boundary of $S$. Thus the lemma is proved.

The next definition is a generalization of one in [1, p. 240].

Definition 1.4. Let $\Sigma$ be a Boolean ring of subsets of a set $S$. The collection of all uniform limits of finite linear combinations of characteristic functions of sets in $\Sigma$ (briefly simple functions) is a Banach algebra and is designated by $B(S, \Sigma)$. The norm in $B(S, \Sigma)$ is given by the formula

$$
\|f\|=\sup _{s \in S}|f(s)|
$$

For a discussion of $B(S, \Sigma)$ spaces see [1].

THEOREM 1.5. Let $I$ be a bounded closed interval in $E^{n}$ and let $R(I)$ be the algebra of Riemann integrable functions on $I$ with $\|f\|$ $=\sup _{x \in I}|f(x)|$. Let $\mathcal{J}$ denote the algebra of Jordan sets in I. Then $R(I)=B(I, \mathcal{J})$.

Proof. Since linear combinations of characteristic functions of $\mathcal{J}$ are in $R(I)$ and $R(I)$ is complete it is clear that $B(I, \jmath) \subset R(I)$. If a function is in both $R(I)$ and $B(I, g)$ its norm is clearly the same in both. We will show that if $f \in R(I)$ then $f \in B(I, \mathcal{J})$.

Let $f \in R(I)$ and consider an arbitrary positive $\epsilon$. We will show that there is an $f_{\epsilon} \in B(I, g)$ such that $\left|f(x)-f_{\epsilon}(x)\right|<\epsilon$ for all $x \in I$.

Using Vitali's covering theorem [2] we can construct a pairwise disjoint collection $V$ of open cubes $v$ in $I$ such that: (1) All except a subset of measure zero of the points of continuity of $f$ are covered; (2) If $v \in v$, and $x_{1}, x_{2} \in v$, then $\left|f\left(x_{1}\right)-f\left(x_{2}\right)\right|<\epsilon / 2$. Let $C=U\{v: v \in \mathcal{U}\}$. Then the following are true: (a) $C$ and $I-C$ are both Jordan sets; (b) Any subset of $I-C$ is a Jordan set; (c) The set theoretic union of any subcollection of $v$ 's in $v$ is a Jordan set. Since $f$ is continuous a.e., then $v$ covers $I$ a.e. We construct a function $f_{c} \in B(I, g)$ as follows: The construction will be broken into two parts $\mathrm{A}$ and B. If $I-C=\varnothing$ we define $f_{A}$ on $I$ by $f_{A}(x)=0$, each $x \in I$, and omit part A. If $I-C \neq \varnothing$ proceed to A.

A. Let $L_{1}=\inf \{f(x): x \in I-C\}$. Define $S_{1}=\{x: x \in I-C$ and $\left.\left|f(x)-L_{1}\right|<\epsilon\right\}$. Form $S_{i}$ inductively as follows for natural numbers $i>1$ : Let $L_{i}=\inf \left\{f(x): x \in I-C\right.$ and $\left.x \notin S_{j}, j<i\right\}$. Define $S_{i}=\left\{x: x \in\left((I-C)-\bigcup_{j<i} S_{j}\right)\right.$ and $\left.\left|f(x)-L_{i}\right|<\epsilon\right\}$. Clearly a finite 
collection $\left\{S_{i}: i=1, \cdots, n\right\}$ will exhaust $I=C . S_{i} \cap S_{j}=\varnothing$ if $i \neq j$ and if $x \in S_{k}$, then $\left|f(x)-L_{k}\right|<\epsilon$. It is also true that each $S_{i} \in \mathcal{g}$. Define $f_{A}=\sum_{\left\{i: S_{i} \neq \phi\right\}} L_{i} \chi_{S_{i}}$ on $I$ where $\chi_{S_{i}}$ is the characteristic function of $S_{i}$. We note that $f_{A} \in B(I, g)$; if $x \in C$, then $f_{A}(x)=0$; and if $x \in I-C$ then $\left|f(x)-f_{A}(x)\right|<\epsilon$.

B. Let $L_{1}^{\prime}=\inf \{f(x): x \in C\}$. Define $v_{L_{1}^{\prime}}=\{v \in v: v$ contains an $\left.x \ni\left|f(x)-L_{1}^{\prime}\right|<\epsilon / 2\right\}$ and define $S_{1}^{\prime}=\bigcup\left\{v: v \in v_{L_{1}^{\prime}}\right\}$. Let $L_{i}^{\prime}=\inf \left\{f(x): x \in C\right.$ and $x \notin S_{j}^{\prime}, j<i$. Define $v_{L_{i}^{\prime}}=\{v: v$ contains an $\left.x \ni\left|f(x)-L_{i}^{\prime}\right|<\epsilon / 2, v \notin V_{L_{i}^{\prime}}, j<i\right\}$. Define $S_{i}^{\prime}=U\left\{v: v \in v_{L_{i}^{\prime}}\right\}$. Clearly a finite collection $\left\{S_{i}^{\prime}: i=1, \cdots, m\right\}$ will exhaust $C$. Each $S_{i}^{\prime} \in \mathcal{g}$ by Lemma 1.3 and $S_{i}^{\prime} \cap S_{j}^{\prime}=\varnothing$ if $i \neq j$. If $x \in S_{i}^{\prime}$, then $\left|f(x)-L_{i}^{\prime}\right|<\epsilon$. We define $f_{B}=\sum_{\left\{i: S_{i}^{\prime} \neq \phi\right\}} L_{i}^{\prime} \chi_{S_{i}^{\prime}}$ on $I$. Then $f_{B}$ $\in B(I, g)$. We observe that if $x \in I-C$, then $f_{B}(x)=0$. If $x \in C$, then $\left|f(x)-f_{B}(x)\right|<\epsilon$.

Define $f_{\epsilon}=f_{A}+f_{B}$. Then $f_{\epsilon} \in B(I, g)$ and for $x \in I,\left|f(x)-f_{\epsilon}(x)\right|<\epsilon$. Since $\epsilon$ is arbitrary it follows that $f \in B(I, g)$.

The characterization of $R(I)$ just given has the following immediate application. A representation of the dual space of $B(S, \Sigma)$ where $\Sigma$ is a Boolean algebra is well known and due independently to Hildebrandt [3] and to Fichtenholz and Kantorovitch [4]. Since $R(I)$ $=B(I, g)$ their result applies here. We content ourselves merely to state the result applied to $R(I)$. The details of the proof can be found in $[1$, p. 258].

Theorem 1.6. Let $B^{*}(I, g)$ designate the dual space of $R(I)=B(I, g)$ and let ba $(I, g)$ denote the space of bounded additive set functions on $\mathrm{J}$. Then there is an isometric isomorphism between $B^{*}(I, \mathfrak{g})$ and $b a(I, g)$ determined by the identity

$$
L^{*} f=\int_{I} f(x) \mu(d x) .
$$

Thus for each $L^{*}$ in $B^{*}(I, g)$ there is a unique $\mu$ in $b a(I, g)$ such that (D) holds; for each $\mu$ in ba $(I, g)$ there is a unique $L^{*}$ such that (D) holds; and the correspondence between $L^{*}$ and $\mu$ is linear and isometric.

2. The algebra $R_{\infty}(I)$. The fact that $g \cap N$ the collection of null Jordan sets is an ideal in $g$ is a well-known obvious observation.

Lemma 2.1. $B(I, g \cap N)$ is a closed ideal in $R(I)$.

Proof. Since by Theorem $1.5 R(I)=B(I, g)$ it suffices to prove that the simple functions in $B(I, g \cap N)$ form an ideal in the set of all simple functions in $B(I, g)$. This is obvious. 
Lemma 2.2. Let $f \in R(I)$. Then $\{x: f(x) \neq 0\} \in N$ if and only if $f \in B(I, g \cap N)$.

Proof. Suppose $\{x: f(x) \neq 0\} \in N$. Let $\epsilon$ be an arbitrary positive number. There is a simple $f_{\epsilon} \in B(I, g)$ such that $\left|f(x)-f_{\epsilon}(x)\right|<\epsilon / 2$, each $x \in I$. Suppose $f_{\mathrm{c}}=\sum_{i=1}^{i=n} A_{i} \chi_{S_{i}}$, where the $S_{i}$ 's are disjoint and elements of $\mathcal{J}$. Form $f_{\epsilon}^{\prime}$ by defining $f_{\epsilon}^{\prime}(x)=0$ on $S_{i}$ if $|f(x)|<\epsilon / 2$ for at least one $x \in S_{i}$. Otherwise define $f_{\epsilon}^{\prime}(x)=f_{\epsilon}(x)$. Then $\left|f(x)-f_{\epsilon}^{\prime}(x)\right|$ $<\epsilon$, for each $x \in I$ and if $f_{\epsilon}^{\prime}(x) \neq 0$ for $x \in S_{j}$ then $f(x) \neq 0$ for $x \in S_{j}$. Hence $m\left(S_{j}\right)=0$. It follows that $f_{\epsilon}^{\prime} \in B(I, g \cap N)$. Conversely suppose $g \in B(I, g \cap N)$. There is a sequence of simple functions $\left\{g_{n}\right\}$ $\subset B(I, g \cap N)$ such that $\left|g(x)-g_{n}(x)\right|<1 / n$, for all $x \in I$. Now $\{x: g(x) \neq 0\} \subset U_{n}\left\{x: g_{n}(x) \neq 0\right\}$. Hence $\{x: g(x) \neq 0\} \in N$.

Definition 2.3. $\|f\|_{\infty^{\prime}}=\inf _{S \in g^{\prime} N} \sup _{x \notin S}|f(x)|$.

Lemma 2.4. Let $f \in R(I)$. Then $\|f\|_{\infty}=\|f\|_{\infty^{\prime}}=\inf _{i \in B(I, g \cap N)}\|f+i\|$.

Proof. We shall show that these norms differ by no more than an arbitrary positive $\epsilon$. By Theorem $1.5, R(I)=B(I, \mathcal{g})$. Hence there is a simple $f_{\epsilon} \in R(I) \ni\left|f_{\epsilon}(x)-f(x)\right|<\epsilon / 2$, for all $x \in I$. It is easy to see that each of the following is true:

$$
\begin{aligned}
\left\|f_{\epsilon}\right\|_{\infty}-\frac{\epsilon}{2} & \leqq\|f\|_{\infty} \leqq\left\|f_{\epsilon}\right\|_{\infty}+\frac{\epsilon}{2}, \\
\left\|f_{\epsilon}\right\|_{\infty^{\prime}}-\frac{\epsilon}{2} & \leqq\|f\|_{\infty^{\prime}} \leqq\left\|f_{\epsilon}\right\|_{\infty^{\prime}}+\frac{\epsilon}{2}, \\
\inf _{i \in B\left(I, g^{\prime} N\right)}\left\|f_{\epsilon}+i\right\|-\frac{\epsilon}{2} & \leqq \inf _{i \in B(I, g \cap N)}\|f+i\| \\
& \leqq \inf _{i \in B\left(I, g^{\prime} N\right)}\left\|f_{\epsilon}+i\right\|+\frac{\epsilon}{2} .
\end{aligned}
$$

Since there clearly is no loss in assuming that $i$ is simple we also easily see the following:

$$
\inf _{i \in B\left(I, g^{\prime} \cap N\right)}\left\|f_{\epsilon}+i\right\|=\left\|f_{\epsilon}\right\|_{\infty^{\prime}}=\left\|f_{\epsilon}\right\|_{\infty} .
$$

The desired result follows at once from (1), (2), (3), and (4).

THEOREM 2.5. Let $R_{\infty}(I)$ have the norm $\|f\|_{\infty}$ and let $R(I) / B(I, g \cap N)$ have the norm $\|f\|^{\prime}=\inf _{i \in B(I, g \cap N)}\|f+i\|$. Then

$$
R_{\infty}(I)=R(I) / B(I, g \cap N) .
$$

Proof. Lemma 2.2 says that $R_{\infty}(I)$ and $R(I) / B(I, g \cap N)$ contain 
the same elements and it is clear that equality of elements is preserved under addition, multiplication and scalar multiplication. Now Lemma 2.4 says the norms are the same. Hence the desired equality is proved.

The following representation of the dual space of $R_{\infty}(I)$ is a special case of a result proved in [6].

Definition 2.6. $b a(I, g \bmod g \cap N)$ is the subspace of $b a(I, g)$ such that $\mu \in b a(I, \mathcal{g} \bmod g \cap N) \leftrightarrow \mu(S)=0$ for each $S \in g \cap N$. The norm of $\mu$ is its $b a(I, g)$ norm.

Theorem 2.7. Let $R_{\infty}^{*}(I)$ designate the dual space of $R_{\infty}(I)$. Then there is an isometric isomorphism between $R_{\infty}^{*}(I)$ and ba $(I, \mathrm{~g} \bmod g \cap N)$ determined by the identity $(\mathrm{D})^{\prime} L^{*}[f]=\int_{I} f(x) \mu(d x)$ where $f$ is any element in $[f]$. Thus for each $L^{*}$ in $R_{\infty}^{*}(I)$ there is a unique $\mu$ in $b a(I, g \bmod g \cap N)$ such that $(\mathrm{D})^{\prime}$ holds; for each $\mu$ in $b a(I, g \bmod g \cap N)$ there is a unique $L^{*}$ such that (D)' holds; and the correspondence between $L^{*}$ and $\mu$ is linear and isometric.

\section{BIBLIOGRAPHY}

1. N. Dunford and Jacob T. Schwartz, Linear operators. I, Interscience, New York, 1958.

2. Henry P. Thielman, Theory of functions of real variables, Prentice-Hall, New York, 1953; pp. 154-155.

3. T. H. Hildebrandt, On bounded functional operations, Trans. Amer. Math. Soc. 36 (1934), 868 875.

4. G. Fichtenholz and L. V. Kantorovitch, Sur les opérations linéaires dans l'espace des fonctions bornées, Studia Math. 5 (1934), 69-98.

5. Charles E. Rickart, General theory of Banach algebras, Van Nostrand, New York, 1960.

6. K. G. Johnson, $B(S, \Sigma)$ algebras, unpublished.

Louisiana State University in New Orleans and TUlane UNIVERSITY OF Loulsiana 